Freiburg, THEP-95/13

gr-qc/9508040

\title{
A EUCLIDEAN BIANCHI MODEL BASED ON $S^{3} / D_{8}^{*}$
}

\author{
Domenico Giulini* \\ Fakultt für Physik, Universität Freiburg \\ Hermann-Herder Strasse 3, D-79104 Freiburg, Germany
}

\begin{abstract}
We explain how the round four sphere can be sliced along homogeneous 3 - manifolds of topology $S^{3} / D_{8}^{*}$. This defines a Euclidean Bianchi type IX model for Einstein's equations with cosmological constant. The geometric properties of this model are investigated.
\end{abstract}

PACS: 02.40.-k, 02.40.Ma, 98.80.Hw

MSC: $53 \mathrm{C} 42$

\section{Introduction}

Spacetimes with homogeneous spatial slices are used extensively as testing ground for Einstein's field equations of general relativity [1-2]. Technically speaking, spatial homogeneity is used to truncate all the infinitely many inhomogeneous degrees of freedom thus keeping only the finitely many homogeneous ones. Analytically the field equations then pose a much simpler problem that can be approached by methods familiar from classical or quantum mechanics. Physically speaking, such a drastic procedure seems justified provided one confines interest to cosmological scales. Classically a spacetime must clearly be a Lorentzian manifold. However, some attempts to quantize gravity make essential use of Euclidean spacetimes, that is, spacetimes with a Riemannian metric. We refer to [3-5] for more details and motivation. The vacuum Einstein equations then simply require the Euclidean

* e-mail: giulini@sun2.ruf.uni-freiburg.de 
spacetime to be an Einstein manifold. For identical reasons as in the Lorentzian case, one is still interested in a homogeneous slicing. Although the specification 'spatial' does not make any sense in the Euclidean context, it is sometimes still used as a reminder on its use for the Lorentzian case $^{1}$. In this paper we consider the Einstein manifold $S^{4}$ with its standard round metric. But instead of slicing it along three dimensional spheres [4] we will slice it along homogeneous manifolds $S^{3} / D_{8}^{*}$. Here $D_{8}^{*}$ is the binary dihedral group of eight elements. As a subgroup of $S U(2)$ it is easily expressed using the unit quaternions $1, i, j, k, i^{2}=j^{2}=k^{2}=-1$, $i j=k$ and cyclic. Then: $D_{8}^{*}=\{ \pm 1, \pm i, \pm j, \pm k\}$. In other words, we consider a Bianchi type IX model [2] with slightly more complicated topology than usual.

We investigate in a self contained fashion the geometry of these isoparametric embeddings, which is indeed quite interesting. The plan of the paper is as follows: In section 1 we show in an explicit and quite elementary fashion how to construct an isometric family of embeddings $S^{3} / D_{8}^{*} \hookrightarrow S^{4}$. In section 2 we determine the corresponding family of 3-dimensional metrics and discuss their geometry. We end with a brief discussion. An appendix provides some background material on the geometry of left invariant metrics on $S^{3} / G$, where $G$ can be any finite subgroup of $S U(2)$. Such geometries are of Bianchi class IX.

\footnotetext{
1 For example, in quantum cosmology one considers manifolds which carry a Riemannian metric on one side of a spatial slice and a Lorentzian metric on the other [5].
} 


\section{Section 1: Isoparametric embeddings of $S^{3} / D_{8}^{*}$ into $S^{4}$}

A one-parameter family of embeddings is called isoparametric, if there is an embedding parameter $t$ that labels the different leaves, such that the extrinsic geometry is homogeneous along a leaf and thus depends only on $t$. Quantities like the principal curvatures or the norm of $d t$ are just functions of $t$. This section deals with the construction of a particular such family of embeddings of a certain 3-manifold into the round unit four sphere.

Let $A$ be an element of $S M_{3}$, the space of real symmetric $3 \times 3$ matrices. If identified with $R^{6}$ via

$$
A=\left(\begin{array}{ccc}
x_{1} & \frac{1}{\sqrt{2}} x_{2} & \frac{1}{\sqrt{2}} x_{3} \\
\frac{1}{\sqrt{2}} x_{2} & x_{4} & \frac{1}{\sqrt{2}} x_{5} \\
\frac{1}{\sqrt{2}} x_{3} & \frac{1}{\sqrt{2}} x_{5} & x_{6}
\end{array}\right)
$$

we see that the two conditions

$$
\begin{aligned}
& \text { 1.) } \operatorname{tr}(A A)=1 \quad R^{6} \rightarrow S^{5} \\
& \text { 2.) } \operatorname{tr}(A)=0 \quad S^{5} \rightarrow S^{4}
\end{aligned}
$$

reduce the manifold of corresponding matrices to the unit five-, respectively unit four sphere. On $R^{6}$ we have the Euclidean metric

$$
d s^{2}=\operatorname{tr}(d A \cdot d A)
$$

where the dot "." indicates matrix- and symmetrized tensor product. It induces the standard metric of the unit four-sphere given by the conditions (1.1) and (1.2).

On $S M_{3}$ there is an action of $S O(3)$ by conjugation

$$
\begin{aligned}
S O(3) \times S M_{3} & \rightarrow S M_{3} \\
(R, A) & \mapsto R A R^{-1}
\end{aligned}
$$

which by (1.1) (1.2) and (1.3) is seen to induce an isometry on $S^{4}$. We are interested in the orbits of this action. Using the fact that the exponential map provides a homeomorphism between the space of symmetric matrices, $S M_{3}$, and the space of positive definite symmetric matrices, $\mathrm{PSM}_{3}$ :

$$
\begin{gathered}
S_{3} \stackrel{\text { homeo }}{\longrightarrow} P S M_{3} \\
A \longmapsto \exp (A)
\end{gathered}
$$


we may think of the eigenvalues as the diameters of an ellipsoid ${ }^{2}$. The group $S O(3)$ then still acts on $\mathrm{PSM}_{3}$ by conjugation leaving invariant the image of $S^{4}$ in $P S M_{3}$ under exp. That these matrices are $S O(3)$-diagonalisable means that we can chose a suitable point on each orbit such that $A$ and hence $\exp (A)$ is diagonal. There are six such points on each generic orbit, due to the permutations of the principal axes generated by $\pi / 2$-rotations about any two different such axes. In order to fix this redundancy, we assume the eigenvalues to be ordered. For later convenience we choose

$$
\lambda_{2} \geq \lambda_{1} \geq \lambda_{3}
$$

If we continue to refer to the general point in $S M_{3}$ as $A$ and the diagonal point as $\Lambda$, we have

$$
\begin{aligned}
\Lambda & =\operatorname{diag}\left\{\lambda_{1}, \lambda_{2}, \lambda_{3}\right\} \\
\exp (\Lambda) & =\operatorname{diag}\left\{e^{\lambda_{1}}, e^{\lambda_{2}}, e^{\lambda_{3}}\right\}
\end{aligned}
$$

We visualize the space of matrices $\mathrm{PSM}_{3}$ as the configuration space of the corresponding ellipsoid with diameters along the principal axes of $e^{\lambda_{1}}, e^{\lambda_{2}}, e^{\lambda_{3}}$. Since the $S O(3)$ action is just the standard rotation of this ellipsoid, we can immediately read off the stabilizer groups. We have two cases corresponding to the principal diameters being pairwise distinct (case 1), or whether two of them, say the first and the second, coincide (case 2). The case where all three coincide is excluded by (1.1) and (1.2).

Case 1: The stabilizer group is generated by $\pi$-rotations about any two of the principal axes. The group thus generated is ${ }^{3}$

$$
Z_{2} \times Z_{2} \cong D_{4}=\{ \pm 1, \pm i, \pm j, \pm k\} / Z_{2}=D_{8}^{*} / Z_{2}
$$

where $1, i, j, k$ denote the unit quaternions. Note that $D_{8}^{*}$ is the pre-image under the identification homomorphism (antipodal map) $\mathcal{A}$

$$
S^{3} \cong S U(2) \stackrel{\mathcal{A}}{\longrightarrow} S O(3) \cong R P^{3}
$$

2 The whole purpose of using the homeomorphic space $P S M_{3}$ is to introduce non-negative diameters and therefore achieve visualization. What really matters is whether the diagonal elements are pairwise distinct or whether two coincide.

$3 D_{2 n}$ is the subgroup of $S O(3)$ corresponding to the symmetries of the n-prism, $D_{4 n}^{*}$ is its 2 -fold cover group in $S U(2)$. The subscript denotes the order of the group. 
The orbit type is therefore given by

$$
S O(3) / D_{4}=S U(2) / D_{8}^{*} \cong S^{3} / D_{8}^{*}
$$

This manifold can be visualized by taking a solid cube whose opposite sides are pairwise identified after a relative $\pi / 2$ - rotation about the axis through their midpoints. It is obvious from the construction that its fundamental group is $D_{8}^{*}$, the higher ones being just those of its covering $S^{3}$. Its homology (resp. cohomology) is $H_{*}=\left(Z, Z_{2} \times Z_{2}, 0, Z\right)\left(\right.$ resp. $\left.H^{*}=\left(Z, 0, Z_{2} \times Z_{2}, Z\right)\right)$.

Case 2: Here the third principal axis is a symmetry axis. The stabilizer group is generated by all rotations about the symmetry axis and a $\pi$-rotation about any other axis perpendicular to it. The orbit manifold can therefore be identified with the configuration space of the symmetry axis, i.e. by the space of all lines through the origin, in other words $R P^{2}$. A more formal way to see this is to embed the acting $\mathrm{SO}(3)$ in $O(3)^{4}$. The stabilizer is then $O(2) \times Z_{2}$ where the $Z_{2}$ is generated by reflections at the origin. $O(2)$ contains reflections about the xz- and yz-plane. Since $Z_{2}$ is normal it acts on the quotient $O(3) / O(2) \cong S^{2}$, just by identifying opposite points. Again we get $R P^{2}$.

Each of the orbits is an isometrically embedded submanifold of $S^{4}$. So we found isometric embeddings of $S^{3}$ resp. $R P^{2}$ into $S^{4}$. The latter one turns out to be congruent to the celebrated Veronese embedding (section 2). If composed with the stereographic projection based at a point in the complement of the orbit in question, we obtain a conformal embedding of $S^{3} / D_{8}^{*}$ resp. $R P^{2}$ into $R^{4}$. We will comment on this at the end of section 2 .

By (1.1) and (1.2), the $\lambda_{i}$ 's just parameterize a great circle of intersection of the 2 -sphere with a plane whose normal is $n=\frac{1}{\sqrt{3}}(1,1,1)$. This normal is obtained from $e_{3}$ by applying the rotation $K=R_{z}(\pi / 4) \circ R_{y}\left(\cos ^{-1}\left(\frac{1}{\sqrt{3}}\right)\right)$ i.e.

$$
K=\left(\begin{array}{ccc}
\frac{1}{\sqrt{6}} & -\frac{1}{\sqrt{2}} & \frac{1}{\sqrt{3}} \\
\frac{1}{\sqrt{6}} & \frac{1}{\sqrt{2}} & \frac{1}{\sqrt{3}} \\
-\sqrt{\frac{2}{3}} & 0 & \frac{1}{\sqrt{3}}
\end{array}\right)
$$

\footnotetext{
4 The point of doing so is that, in contrast to $S O(3)$, the stabilizer group is now the product of subgroups of $O(3)$.
} 
In the rotated frame, $e_{i}^{\prime}=K_{i}^{j} e_{j}$, the great circle has the coordinate representation $\vec{x}(t)=(\cos t, \sin t, 0)$ so that we find

$$
\begin{aligned}
& \lambda_{1}(t)=\sqrt{\frac{2}{3}} \cos \left(t+\frac{\pi}{3}\right) \\
& \lambda_{2}(t)=\sqrt{\frac{2}{3}} \cos \left(t-\frac{\pi}{3}\right) \\
& \lambda_{3}(t)=\sqrt{\frac{2}{3}} \cos (t+\pi) .
\end{aligned}
$$

For later application we also note:

$$
\begin{aligned}
& \lambda_{1}-\lambda_{2}=-\sqrt{2} \sin t \\
& \lambda_{2}-\lambda_{3}=-\sqrt{2} \sin \left(t-\frac{2}{3} \pi\right) \\
& \lambda_{3}-\lambda_{1}=-\sqrt{2} \sin \left(t+\frac{2}{3} \pi\right) .
\end{aligned}
$$

Condition (1.5) then restricts the range of $t$ to $t \in[0, \pi / 3]$ where interior points correspond to generic orbits of topology $S^{3} / D_{8}^{*}$ and boundary points $(t=0, t=\pi / 3)$ to degenerate orbits of topology $R P^{2}$. In the sequel we set $S^{3} / D_{8}^{*}=: \Sigma$. We have thus found a one parameter family of embeddings

$$
\begin{gathered}
\left.\phi_{t}: \Sigma \longrightarrow S^{4} \quad t \in\right] 0, \pi / 3[ \\
\Sigma \longmapsto \Sigma_{t} \subset S^{4}
\end{gathered}
$$

where $\Sigma_{t}$ denotes the image of $\Sigma$ under $\phi_{t}$ in $S^{4}$.

$\Sigma$ is only a special example of a so called space form, which are defined to be the quotient spaces of $S^{3}$ with respect to a freely acting finite subgroup $G$ of $S O(4)$. Its action on $S^{3}$ is best understood using

$$
S O(4) \cong \frac{S U(2) \times S U(2)}{Z_{2}}
$$

where the $Z_{2}$ is generated by $(-1,-1)$. Elements in $S O(4)$ are then written as $Z_{2}$-equivalence classes $[g, h]$ with $g, h \in S U(2)$. If we then identify $S^{3}$ with $S U(2)$, the action is given by:

$$
\begin{aligned}
S^{3} \times S O(4) & \longrightarrow S^{3} \\
\{p,[g, h]\} & \longmapsto g \cdot p \cdot h^{-1}
\end{aligned}
$$


Equation (1.15) suggests to call $[ \pm 1, S U(2)]=: S U(2)_{R}$ the right $S U(2)$ and $[S U(2), \pm 1]=: S U(2)_{L}$ the left $S U(2)$. Clearly $S U(2)_{R} \cap S U(2)_{L}=\{[1,1],[1,-1]\}$. If $G$, besides being a finite subgroup of $S O(4)$, is also a non-cyclic (i.e. not a $Z_{p}$ ) finite subgroup of $S U(2)$ it must sit in either $S U(2)_{R}$ or $S U(2)_{L}$ in order to act freely on $S^{3}$. In these cases the resulting quotient space is a homogeneous space form and $G$ can w.l.o.g. be taken as a subgroup of $S U(2)_{R}{ }^{5}$. If we started with the standard round metric on $S^{3}$ (the bi-invariant metric on $S U(2)$ ), the residual isometry group of $S^{3} / G$ would be

$$
\operatorname{Isom}\left(S^{3} / G\right)=S O(3) \times N_{G}(S U(2)) / G
$$

where $N_{G}(S U(2))$ denotes the normalizer of $G$ in $S U(2)$. All these spherical space forms can be obtained by suitably identifying opposite sides of some polyhedron (the fundamental domain). It is not hard to show that the symmetry group of this polyhedron is just the normalizer of $G / Z_{2}$ (the image of $G$ under the quotient map $\mathcal{A}$ of eq. (1.9)) in the diagonal $S O(3)$ of $S O(4)$. In the case at hand, $G=D_{8}^{*}$ and we already noted that the fundamental domain for $\Sigma$ is a cube. We have $N_{D_{8}^{*}}(S U(2))=O^{*}$ and hence

$$
\operatorname{Isom}(\Sigma)=S O(3) \times P_{3}
$$

where $P_{3}$ is the six element permutation group of three objects. The diagonal $P_{3}$ in Isom $(\Sigma)$ can then be identified with the permutations of the principle axes of the ellipsoid in the previous discussion.

Clearly, we can not expect the orbits $\Sigma_{t}$ to have this maximally symmetric metric, albeit we know by construction that the isometry group must contain a left acting $\mathrm{SO}(3)$. There is however no reason for the $\pi / 2$-rotations about principal axes to be isometries as well. As a minimal symmetry we therefore expect the generic orbits to have a metric that, if lifted to $S^{3}(\cong S U(2))$, is left invariant and whose right isometries are equal to $D_{8}^{*}$. The next section contains an explicit calculation of the orbit metric showing that the metric is indeed of the minimal symmetric form.

Finally we make some topological remarks. Since the unit quaternion $k$ (say) generates a normal $Z_{4}$-subgroup of $D_{8}^{*}$, we can take the quotient $S^{3} \rightarrow \Sigma$ stepwise;

${ }^{5}$ If we call $\Sigma_{R}$ (resp. $\Sigma_{L}$ ) the quotient manifold with respect to the right (resp. left) $S U(2)$ action with points $[p]_{R}\left(\right.$ resp. $\left.[p]_{L}\right), p \in S^{3}$, then they are diffeomorphically related by $[p]_{R} \mapsto\left[p^{-1}\right]_{L}$ 
first with respect to the $Z_{4}$, then dividing the remaining quotient $Z_{2}$. After the first step we arrive at the lens space $L(4,1)$ with its well known structure as a principal $U(1)$-bundle over $S^{2}$. The next step transforms this into a circle bundle over $R P^{2}$, which is clearly not a principal bundle since the (right) $U(1)$ which acted on $L(4,1)$ does not act on the $Z_{2}$-quotient of it. $\Sigma$ is thus a circle bundle over $R P^{2}$, and if we cut $S^{4}$ along a $\Sigma_{t}$ each piece is a 2 -disc bundle over $R P^{2}$ where the zero section can be identified with Veronese's $R P^{2}$ in $S^{4}$.

\section{Section 2. The metric}

Let us compute the metric by coordinatizing the orbit direction by the group parameters and the perpendicular direction by the single parameter that labels the range of the eigenvalues $\lambda_{1}, \lambda_{2}, \lambda_{3}$ obeying (1.1), (1.2) and (1.5). We thus write

$$
A=R \Lambda R^{-1}
$$

This implies that our $S O(3)$-action is given by left translations on the $R$-factor, whereas rotations of the principal axes (by which the identification $S O(3) \rightarrow \Sigma$ is made) correspond to right translations. A short calculation then leads to

$$
d s^{2}=\operatorname{tr}(d A \cdot d A)=\operatorname{tr}\left\{d \Lambda \cdot d \Lambda+2\left[\left(R^{-1} d R \Lambda\right)^{2}-\left(R^{-1} d R\right)^{2} \Lambda^{2}\right]\right\}
$$

Introducing the basis ${ }^{6}$ of left invariant 1-forms via

$$
R^{-1} d R=: T^{a} l_{a}
$$

where the basis of $s o(3)$, the Lie algebra of $S O(3)$, is given by

$$
\left(T^{a}\right)_{c}^{b}=\varepsilon_{a b c}, \quad \text { s.t. } \quad\left[T^{a}, T^{b}\right]=-\varepsilon_{a b c} T^{c}
$$

\footnotetext{
6 Whereas right invariant sections over $S O(3)$ induce well defined sections on $\Sigma$, this holds in the left invariant case only for even-valence tensors, like e.g. the metric (2.8). The odd-valence sections are well defined only locally, and globally up to sign. In the sequel we still refer to the basis of left invariant 1-forms, or their dual basis of left invariant vector fields without explicit mention of this sign ambiguity. Note that the notion of integral lines of left invariant vector fields still makes unambiguous sense.
} 
one explicitly finds, using Euler angles $(\psi, \theta, \varphi)$,

$$
\begin{aligned}
l_{1} & =\cos \psi \sin \theta d \varphi-\sin \psi d \theta \\
l_{2} & =\sin \psi \sin \theta d \varphi+\cos \psi d \theta \\
l_{3} & =d \psi+\cos \theta d \varphi \\
\text { obeying } \quad d l_{a} & =\frac{1}{2} \varepsilon_{a b c} l_{b} \wedge l_{c} .
\end{aligned}
$$

Inserting (1.6) and (2.3) into (2.2) gives:

$$
d s^{2}=\left(d \lambda_{1}^{2}+d \lambda_{2}^{2}+d \lambda_{3}^{2}\right)+2\left[\left(\lambda_{2}-\lambda_{3}\right)^{2} l_{1}^{2}+\left(\lambda_{3}-\lambda_{1}\right)^{2} l_{2}^{2}+\left(\lambda_{1}-\lambda_{2}\right)^{2} l_{3}^{2}\right]
$$

Using (1.12) and (1.13) we get the final form of the metric:

$$
d s^{2}=d t^{2}+4\left[\sin ^{2}\left(t-\frac{2}{3} \pi\right) l_{1}^{2}+\sin ^{2}\left(t+\frac{2}{3} \pi\right) l_{2}^{2}+\sin ^{2}(t) l_{3}^{2}\right]
$$

For small values of $t$ this expression becomes:

$$
d s^{2}=d t^{2}+4 t^{2} l_{3}^{2}+3\left(d \theta^{2}+\sin ^{2} \theta d \varphi^{2}\right)
$$

The regularity at $t=0$, though obvious from the whole geometry, is now explicitly seen due to the identification via the right $\exp \left(T_{3} \pi\right)$ translation ${ }^{7}$. More importantly, the degenerated orbit, $R P^{2}$, is seen to carry a metric of constant sectional curvature $1 / 3$ and must therefore be congruent to the Veronese embedding. Clearly, all this holds equally well for $t=\frac{\pi}{3}$.

Let us now focus on the generic orbits. On the universal covering, $S^{3}$, the Euler angles have the range $0 \leq \psi \leq 4 \pi, 0 \leq \theta \leq \pi$ and $0 \leq \varphi \leq 2 \pi$. A typical orbit will be covered eight times. The volume of the typical orbit then follows:

$$
\begin{aligned}
V & =\left|\sin (t) \sin \left(t-\frac{2}{3} \pi\right) \sin \left(t+\frac{2}{3} \pi\right)\right| \int_{S^{3}} l_{1} \wedge l_{2} \wedge l_{3} \\
& =\left|\sin (t) \sin \left(t-\frac{2}{3} \pi\right) \sin \left(t+\frac{2}{3} \pi\right)\right| 8\left(2 \pi^{2}\right) \\
& =\sin (t)\left[\cos ^{2}(t)-\frac{1}{4}\right] 8\left(2 \pi^{2}\right)=\sin (t)\left[\frac{3}{4}-\sin ^{2}(t)\right] 8\left(2 \pi^{2}\right) \\
& =\left|\lambda_{1}-\lambda_{2}\right|\left|\lambda_{2}-\lambda_{3}\right|\left|\lambda_{3}-\lambda_{1}\right| 2 \sqrt{2}\left(2 \pi^{2}\right)
\end{aligned}
$$

\footnotetext{
7 Right translations are generated by left invariant vector fields. The left invariant vector field corresponding to $T_{3}$ is $\frac{\partial}{\partial \psi}$ hence $\psi$ has range $0 \leq \psi \leq \pi$
} 
The maximal value is reached for $t=\frac{\pi}{6}$ and given by

$$
V_{\max }^{(3)}=4 \pi^{2}
$$

which is twice the volume of the equatorial 3-sphere in the unit $S^{4}$. As a check, we calculate the total 4-dimensional volume from (2.8) and find

$$
\begin{aligned}
V^{(4)} & =16 \pi^{2} \int_{0}^{\frac{\pi}{3}} d t \sin (t)\left[\cos ^{2} t-\frac{1}{4}\right] \\
& =16 \pi^{2} \int_{\frac{1}{2}}^{1} d z\left[z^{2}-\frac{1}{4}\right]=\frac{8}{3} \pi^{2}
\end{aligned}
$$

which is just the volume of the unit 4-sphere as it must be. Although (2.11) is just the maximal volume amongst the particular 1-parameter variation (2.9), general $\operatorname{arguments}^{8}$ imply stationarity amongst all variations and hence that the image of the $t=\pi / 6$ embedding of $S^{3} / D_{8}^{*}$ being a minimal ${ }^{9}$ submanifold. We can see this directly by computing the extrinsic curvature tensor $K=K_{a b} \theta^{a} \otimes \theta^{b}$ in the orthogonal frame

$$
\left\{\theta^{a}\right\}=\left\{d t, 2 \sin \left(t-\frac{2}{3} \pi\right) l_{1}, 2 \sin \left(t+\frac{2}{3} \pi\right) l_{2}, 2 \sin (t) l_{3}\right\}
$$

The result is

$$
\begin{aligned}
\left\{K_{a b}(t)\right\} & =\operatorname{diag}\left\{\cot \left(t-\frac{2}{3} \pi\right), \cot \left(t+\frac{2}{3} \pi\right), \cot (t)\right\} \\
\operatorname{tr}\left\{K_{a b}(t)\right\} & =3 \cot (t)\left[\frac{\cot ^{2}(t)-3}{3 \cot ^{2}(t)-1}\right] .
\end{aligned}
$$

8 The argument runs as follows: Let $K$ be the trace of the extrinsic curvature tensor, and $\nu$ the normal vector field to the embeddings $\phi_{t}$. Fix $t_{1}$ such that $\phi_{t_{1}}(\Sigma)=\Sigma_{t_{1}}$ is a generic orbit. Define a section $\eta$ in the normal bundle of $\Sigma_{t_{1}}$ by $\eta=K \nu$. Since $G$ acts as isometries, we have $g_{*} \eta=\eta \forall g \in G$ and hence $g \cdot \exp _{m}(s \eta)=\exp _{g \cdot m}(s \eta)$ for sufficiently small parameters $s$ and $\forall m \in \Sigma_{t_{1}}$. Nearby orbits are therefore labeled by $s$. Using $\eta$ as the deformation field in the first variation formula of the area, we obtain:

$$
\left.\frac{d}{d s}\right|_{s=0} A(s)=-\int_{\Sigma^{*}} K\langle\nu, \eta\rangle \omega=-\int_{\Sigma^{*}} K^{2} \omega
$$

We conclude that stationarity with respect to the embedding parameter $s$ implies $K=0$

9 According to standard terminology, a stationary point of the area functional is called a minimal surface and a stable minimal surface if it is a true minimum. 
Note that the principal curvature directions (eigenvectors of $K_{a}^{b}$ ) are just the left invariant vector fields, dual to the 1-form basis (2.12), which, using (2.8), are readily seen ${ }^{10}$ to be geodesic on $S^{4}$. Their integral curves are thus great circles of $S^{4}$.

(2.13) implies $\left\{K_{a b}\left(\frac{\pi}{6}\right)\right\}=\operatorname{diag}(0, \sqrt{3},-\sqrt{3})$ so that $\operatorname{tr}\left\{K_{a b}\right\}\left(\frac{\pi}{6}\right)=0$ which means that $\Sigma_{\frac{\pi}{6}}$ is a minimal, non totally geodesic submanifold of $S^{4}$.

Although there is no isometry of $S^{4}$ inducing the identity on $\Sigma_{\frac{\pi}{6}}$, there is an isometry exchanging the two halves to either side of $\Sigma_{\frac{\pi}{6}}$ orbitwise while inducing a non-trivial isometry on $\Sigma_{\frac{\pi}{6}}$. It is given by the standard antipodal map $\mathcal{A}$ of $S^{4}$. To see this, first consider the map

$$
\begin{aligned}
\sigma:[0, \pi / 3] & \longrightarrow[0, \pi / 3] \\
t & \longmapsto \frac{\pi}{3}-t
\end{aligned}
$$

whose action on the functions $\lambda_{i}$ in (1.12) is just given by.

$$
\begin{aligned}
& \lambda_{1} \circ \sigma=-\lambda_{1} \\
& \lambda_{2} \circ \sigma=-\lambda_{3} \\
& \lambda_{3} \circ \sigma=-\lambda_{2}
\end{aligned}
$$

On the other hand, consider the following right multiplication map ${ }^{11}$

$$
\begin{aligned}
R_{\exp \left(-T_{1} \pi / 2\right)}: \Sigma & \longrightarrow \Sigma \\
{[p] } & \longmapsto\left[p \cdot \exp \left(-T_{1} \pi / 2\right)\right]
\end{aligned}
$$

where for $p \in S^{3},[p] \in \Sigma$ denotes the equivalence class w.r.t. right multiplications by $D_{8}^{*}$. As is easily seen from (2.1) it acts on $A$ by just exchanging the second and third eigenvalue of $\Lambda$. The composition of (2.15) with (2.17) then acts on $A$ by multiplication with -1 , which is just the antipodal map. It clearly forms an isometry of (2.8). This means that $\sum_{\frac{\pi}{6}}$ splits $S^{4}$ into two isometric halves. Looking at eq.

10 Standard formulae for the Levi-Civita connection imply: $\left(\nabla_{X} X, Y\right)=X(X, Y)-\frac{1}{2} Y|X|^{2}-$ $(X,[X, Y])$. Taking $X$ to be left invariant gives trivially zero for all left invariant $Y$ and zero for $Y=\frac{\partial}{\partial t}$ where the first term is zero and the second and third just cancel. Hence $\nabla_{X} X=0$.

11 Note that since $\exp \left(-T_{1} \pi / 2\right) \in N_{D_{8}^{*}}(S U(2))$ this is a well defined map on the quotient $\Sigma$. 
(2.8) we see that for $t \in] 0, \pi / 3$ [ the only point where two of the prefactors of the $l_{i}$ 's coincide is $t=\pi / 6$. This implies that on a general $\Sigma_{t}$, the only isometries are the left $S O(3)$ actions, whereas for $t=\pi / 6$ there is an additional $Z_{2}$ factor generated by the restriction of $\mathcal{A}$. This should be compared with eq. (1.18). Only a $Z_{2}$ subgroup of $P_{3}$ survived. Explicitly the metric on $\Sigma_{\frac{\pi}{6}}$ is given by

$$
d s^{2}=4 l_{1}^{2}+l_{2}^{2}+l_{3}^{2}
$$

On $S^{3}$ it has a $S U(2)$-left $U(1)$-right isometry group. On the quotient space, $\Sigma$, this reduces to the $S O(3)$-left $Z_{2}$-right isometries.

In the appendix it is shown that a general left invariant metric $d s^{2}=\tau_{1}^{2} l_{1}^{2}+$ $\tau_{2}^{2} l_{2}^{2}+\tau_{3}^{2} l_{3}^{2}$ has vanishing Ricci-scalar if and only if $\tau_{i}=\tau_{j}+\tau_{k}$ for some cyclic permutation $i, j, k$ of 1.2.3. But from (2.7) it is immediate that this is the case for all $\Sigma_{t}$. Note that since (2.8) is the metric on the unit four sphere, it satisfies

$$
R_{\mu \nu}^{(4)}=\Lambda g_{\mu \nu} \quad \text { where } \quad \Lambda=3 .
$$

Performing a $(3+1)$ - decomposition for the purely normal components of the Ricci-tensor with respect to the hypersurfaces $\Sigma_{t}$, one obtains the corresponding Gauss-Codazzi equations (Hamiltonian constraint)

$$
\left.\operatorname{tr}\left(K^{2}(t)\right)+(\operatorname{tr}(K(t)))^{2}=2 \Lambda-R^{(3)}(t)=6 \quad \forall t \in\right] 0, \frac{\pi}{3}[.
$$

where the last identity can also be explicitly checked from (2.13) using standard trigonometric identities and $\Lambda=3$. In general relativity the left hand side of (2.20) is just the so called kinetic term, which, except for its non positive definite nature, formally represents the kinetic energy per unit volume in the Hamiltonian. The 'motion' described by $t \mapsto \Sigma_{t}$ thus has 'kinetic' energy proportional to the volume $(2.9)$.

Let us finally have a look at Einstein's action principle. The field equations (2.19) can be derived from the action functional

$$
S=\int_{M}\left(R^{(4)}-2 \Lambda\right) \mu_{M}+2 \int_{\partial M} K_{a}^{a} \mu_{\partial M}
$$

where $R^{(4)}$ is the 4-dimensional Ricci-scalar and $\mu$ the standard measure induced by the metric. The boundary term is necessary since the Ricci-scalar contains second 
derivatives [3]. Let us now calculate the action for a solution if $M$ is just half the 4 -sphere, cut along a minimal hypersurface $\Sigma=\partial M$. The action for a solution is then just given by the integral of $\frac{1}{2} R^{(4)}$ over $M$, which leads to $S=8 \pi^{2} r^{2}$, where $r$ is the radius of the 4 -sphere. The boundary term in (2.21) does not contribute due to $\Sigma$ being minimal. In particular, if $V$ denotes the volume of $\Sigma$, the action is proportional to $V^{\frac{2}{3}}$. We can use this to compare the actions for different $\Sigma$ of equal 3-volume. Specifically, we compare the following two cases: 1.) $\Sigma_{(1)}$ is the equatorial 3-sphere with volume $V_{(1)}=2 \pi^{2} r_{(1)}^{3}, 2$.) $\Sigma_{(2)}=\Sigma_{\frac{\pi}{6}}$, as constructed above, with volume $V_{(2)}=4 \pi^{2} r_{(2)}^{3}$ (compare $(2.11)$ ). Since we want $V_{(1)}=V_{(2)}$ we must have $r_{(2)}=2^{-\frac{1}{3}} r_{(1)}$ and hence for the actions:

$$
S_{(2)}=2^{-\frac{2}{3}} S_{(1)}=2^{-\frac{4}{3}} 8(\pi V)^{\frac{2}{3}}
$$

For fixed 3-volume, the "creation" of the manifold $S^{3} / D_{8}^{*} \operatorname{costs}$ only $2^{-\frac{2}{3}}$ times as much action as the "creation" of a round 3 -sphere.

\section{Appendix}

In this appendix we collect some formulae concerning the geometry of the general left invariant metric

$$
d s^{2}=\tau_{1}^{2} l_{1}^{2}+\tau_{2}^{2} l_{2}^{2}+\tau_{3}^{2} l_{3}^{2}=\omega_{1}^{2}+\omega_{2}^{2}+\omega_{3}^{2}
$$

where $\left\{\tau_{1}, \tau_{2}, \tau_{3}\right\}$ is the standard left invariant basis as in (2.5) which obeys (2.6), and $\omega_{i}=\tau_{i} l_{i}$ (no summation). The normalization is such that the integral over the unit 3-sphere of $l_{1} \wedge l_{2} \wedge l_{3}$ is $16 \pi^{2}$. This is easily verified using (2.5) (note that $0 \leq \psi \leq 4 \pi)$. Solving Cartan's first structure equation $d \omega_{a}+\omega_{a b} \wedge \omega_{b}=0$ for the antisymmetric connection 1-forms $\omega_{a b}=-\omega_{b a}$ yields

$$
\omega_{12}=\eta_{3} \omega_{3} \quad \text { and cyclic }
$$

where 'and cyclic' refers to any cyclic permutation of $1,2,3$, and

$$
\eta_{1}=\frac{1}{2}\left(-\frac{\tau_{1}}{\tau_{2} \tau_{3}}+\frac{\tau_{2}}{\tau_{3} \tau_{1}}+\frac{\tau_{3}}{\tau_{1} \tau_{2}}\right) \quad \text { and cyclic }
$$


The curvature 2-form $\Omega_{a b}=d \omega_{a b}+\omega_{a c} \wedge \omega_{c b}$ has then the non-vanishing components

$$
\Omega_{12}=\left(-\eta_{1} \eta_{2}+\eta_{2} \eta_{3}+\eta_{3} \eta_{1}\right) \omega_{1} \wedge \omega_{2}=\frac{1}{2} R_{12 a b} \omega_{a} \wedge \omega_{b} \quad \text { and cyclic }
$$

The non-vanishing components of the Ricci-tensor are then the diagonal ones:

$$
\begin{aligned}
R_{11} & =R_{2121}+R_{3131}=2 \eta_{2} \eta_{3} \quad \text { and cyclic } \\
& =\frac{1}{2\left(\tau_{1} \tau_{2} \tau_{3}\right)^{2}}\left(\tau_{1}^{4}-\left(\tau_{2}^{2}-\tau_{3}^{2}\right)^{2}\right) \quad \text { and cyclic }
\end{aligned}
$$

which leads to the Ricci-scalar

$$
\begin{aligned}
R & =R_{11}+R_{22}+R_{33}=2\left(\eta_{1} \eta_{2}+\eta_{2} \eta_{3}+\eta_{3} \eta_{1}\right) \\
& =\frac{1}{\left(\tau_{1} \tau_{2} \tau_{3}\right)^{2}}\left(-\left(\tau_{1}^{2}-\tau_{2}^{2}-\tau_{3}^{2}\right)+4 \tau_{2}^{2} \tau_{3}^{2}\right) \quad \text { or cyclic } .
\end{aligned}
$$

In 3 dimensions the Ricci-tensor and Ricci-scalar determine the curvature. According to the last two expressions it takes the simple form:

$$
\Omega_{12}=\left(\frac{1}{2} R-R_{33}\right) \omega_{1} \wedge \omega_{2} \quad \text { and cyclic }
$$

Observation. Since no two of the $\eta_{i}$ can simultaneously vanish, at most one of the diagonal Ricci-components $R_{11}, R_{22}, R_{33}$ can be zero. E.g. $R_{11}=0 \Leftrightarrow \tau_{2}^{2}=\tau_{1}^{2}+\tau_{3}^{2}$ or (exclusively) $\tau_{3}^{2}=\tau_{1}^{2}+\tau_{2}^{2}$. The Ricci-scalar vanishes, if and only if $\tau_{a}=\tau_{b}+\tau_{c}$ for some cyclic permutation $a, b, c$ of $1,2,3$. If $R=0$, the Ricci-tensor is non-degenerate and determines the sectional curvatures via $R_{1212}=-R_{33}$ and cyclic.

Let us briefly consider the case $R=0$. Without loss of generality we assume $\tau_{1}+\tau_{2}=\tau_{3}$. Then the non-vanishing components for the Ricci-tensor and sectional curvature are:

$$
\begin{aligned}
& R_{11}=-R_{2323}=\frac{-2}{\tau_{2}\left(\tau_{1}+\tau_{2}\right)} \\
& R_{22}=-R_{1313}=\frac{-2}{\tau_{1}\left(\tau_{1}+\tau_{2}\right)} \\
& R_{33}=-R_{1212}=\frac{2}{\tau_{1} \tau_{2}} .
\end{aligned}
$$

\section{Acknowledgements}

I thank Gary Gibbons for initiating this study and useful discussions. 


\section{References}

[1] General Relativity, An Einstein Centenary Survey. Edited by S.W. Hawking and W. Israel. Cambridge University Press, 1979.

[2] M.A.H. MacCallum: Anisotropic and Inhomogeneous Relativistic Cosmology. In $[1]$.

[3] S.W. Hawking: The Path Integral Approach to Quantum Gravity. In [1].

[4] S.W. Hawking (1984). The Quantum State of the Universe. Nucl. Phys. B 239: 257-276.

[5] G.W. Gibbons, J. Hartle (1991). Real Tunneling Geometries and the Large-Scale Topology of the Universe. Phys. Rev. D, 42, 2458. 